\title{
nature protocols
}

\section{Author Correction: Efficient genetic engineering of human intestinal organoids using electroporation}

Masayuki Fujii, Mami Matano, Kosaku Nanki and Toshiro Sato

Correction to: Nature Protocols https://doi.org/10.1038/nprot.2015.088, published online 3 September 2015

The version of this paper originally published shows incorrect units for two plasmid concentrations. In the "Reagent Setup" section, the instructions for sgRNA-Cas9 plasmid should read "Adjust the concentration of each plasmid to $1 \mu \mathrm{g} \mu \mathrm{l}^{-1}$," rather than "to $1 \mu \mathrm{g} \mathrm{ml}^{-1}$." Similarly, all concentrations in the tables in Steps 49A, 49C, and 49D should be in $\mu \mathrm{g} \mu^{-1}$ instead of $\mu \mathrm{g} \mathrm{ml}^{-1}$. Please note that these units have not been corrected in the PDF and HTML versions of the protocol available online.

Published online: 5 December 2018

https://doi.org/10.1038/s41596-018-0107-5

\section{Author Correction: Design, execution, and analysis of CRISPR-Cas9-based deletions and genetic interaction networks in the fungal pathogen Candida albicans}

\author{
Viola Halder, Caroline B. M. Porter, Alejandro Chavez (D), Rebecca S. Shapiro (D) \\ Correction to: Nature Protocols https://doi.org/10.1038/s41596-018-0122-6, published online 8 February 2019
}

The version of this paper originally published contained reference errors. The sentence "To dissect complex genetic interactions in C. albicans, a CRISPR-Cas9-based Gene Drive Array (GDA) was developed" incorrectly cited ref. 13, and should have cited ref. 14. In addition, the reference included as ref. 13 in the original paper was incorrect, and should have been the following: Shapiro, R. S., Chavez, A. \& Collins, J. J. CRISPR-based genomic tools for the manipulation of genetically intractable microorganisms. Nat. Rev. Microbiol. 16, 333-339 (2018). This reference should have been cited after the sentence "Recent innovations in CRISPR-Cas9-based genome editing have facilitated such genetic interaction analyses." The original reference 13 (Gerami-Nejad, M., Zacchi, L. F., McClellan, M., Matter, K. \& Berman, J. Shuttle vectors for facile gap repair cloning and integration into a neutral locus in Candida albicans. Microbiology 159, 565-579 (2013)) should have been cited later in the paper, and is now in the reference list as ref. 27. As a result, original references $27-33$ have been renumbered in the reference list and in the text. These changes have been made in the PDF and HTML versions of the protocol.

Published online: 11 April 2019

https://doi.org/10.1038/s41596-019-0157-3

\section{Author Correction: Using the tube test to measure social hierarchy in mice}

\section{Zhengxiao Fan (D), Hong Zhu (D), Tingting Zhou (D), Sheng Wang, Yan Wu (D) Hailan Hu (D)}

Correction to: Nature Protocols https://doi.org/10.1038/s41596-018-0116-4, published online 15 February 2019

In the version of this paper originally published, an affiliation for Zhengxiao Fan was omitted. In addition, the Reporting Summary incorrectly indicated that human research participants had been used in the study, instead of animal subjects. These errors have been corrected in the PDF and HTML versions of the protocol.

Published online: 11 April 2019

https://doi.org/10.1038/s41596-019-0158-2 